Círculo de Lingüística Aplicada a la Comunicación ISSN: 1576-4737

\title{
WoSSP 2019 16TH Workshop on Syntax, Semantics and Phonology
}

https://eventos.ucm.es/27328/section/15791/16th-workshop-on-syntax-semantics-andphonology.html

\section{Call for papers}

This workshop is aimed at graduate students. It will held at the Universidad Complutense de Madrid on 17-18, 2019. Abstracts are encouraged on any aspect of theoretical and applied linguistics, especially -but not exclusively- semantics, syntax, lexicology, interfaces, morphology, phonology, clinical and computational linguistics. Authors cannot hold a PhD before June 2019. The deadline is February 9, 2019 and submissions are limited to a maximum of one individual and one joint abstract, or two joint abstracts per author. Accepted papers will be notified on April. A limited number of abstracts will also be accepted for a poster session.

\section{Invited speakers}

Antonio Fábregas Alfaro (Universidad de Tromsø)

Violeta Martínez-Paricio (Universitat de València)

Michelle Sheehan (University of Anglia Ruskin )

\section{Scientific committee}

Maria Auxiliadora Barrios (UCM), Nuria Bel (UPF), Teresa Cabré (UAB), Bruno Camus (UCLM), Maria Ángeles Carrasco (UCLM), Jan Casalicchio (University of Utrecht), Elena Castroviejo (UPV), Roberta D'Alessandro (UIL/OTS), Maia Duguine (Iker-CNRS), Luis Eguren (UAM), Victoria Escandell-Vidal (UNED), María Teresa Espinal (UAB), Urtzi Etxeberria (Iker-CNRS), Antonio Fábregas (University of Tromsø), Ana María Fdez. Pampillón (UCM), Ángel Gallego (UAB), Luis García Fernández (UCM), Irene Gil (Centro de Estudios de la RAE), Silvia Gumiel (UAH), María Edita Gutiérrez (UCLM), Antonio Hidalgo (UV), Aritz Irurtzun (Iker-CNRS), Ángel Jiménez (US), José María Lahoz (UCM), Manuel Leonetti (UAH/UCM), Joan Mascaró (UAB), Antonio Moreno Sandoval (UAM), Elena del Olmo (UCM), Javier Ormazábal (UPV), Dennis Ott (University of Ottawa), Nuria Polo (UNED), Clàudia Pons (UB), Julio Villa-García (University of Manchester), Cristina Sánchez López (UCM), Mariona Taulé (UB), Francesc Torres-Tamarit (CNRS-U. Paris), Margarita Vivanco (UCLM). 